\title{
Design of a de novo aggregating antimicrobial peptide and a bacterial conjugation-based delivery system
}

\author{
by \\ Logan Thrasher Collins \\ Department of Molecular, Cellular, and Developmental Biology; University of Colorado at \\ Boulder
}

Thesis advisor:

Dr. Anushree Chatterjee; Department of Chemical and Biological Engineering

Defense committee:

Dr. Anushree Chatterjee; Department of Chemical and Biological Engineering

Dr. Jennifer Knight; Department of Molecular, Cellular, and Developmental Biology

Dr. Jennifer Martin; Department of Molecular, Cellular, and Developmental Biology 


\begin{abstract}
Antibacterial resistance necessitates the development of novel treatment methods for infections. Protein aggregates have recently been applied as antimicrobials to disrupt bacterial homeostasis. Past work on protein aggregates has focused on genome mining for aggregationprone sequences in bacterial genomes rather than on rational design of aggregating antimicrobial peptides. Here, we use a synthetic biology approach to design an artificial gene encoding a de novo aggregating antimicrobial peptide. This artificial gene, opaL (overexpressed protein aggregator Lipophilic), disrupts bacterial homeostasis by expressing extremely hydrophobic peptides. When this hydrophobic sequence is disrupted by acidic residues, consequent aggregation and antimicrobial effect decreases. Further, we developed a probiotic delivery system using the broadhost range conjugative plasmid RK2 to transfer the gene from donor to recipient bacteria. We utilize RK2 to mobilize a shuttle plasmid carrying opaL by adding the RK2 origin of transfer. We show that opaL is non-toxic to the donor, allowing for maintenance and transfer since its expression is under control of a promoter with a recipient-specific T7 RNA polymerase. Upon mating of donor and recipient Escherichia coli, we observe selective growth repression in T7 polymerase-expressing recipients. This technique could be used to target desired pathogens by selecting pathogen-specific promoters to control T7 RNA polymerase expression and provides a basis for the design and delivery of aggregating antimicrobial peptides.
\end{abstract}




\section{Introduction}

Antibacterial resistance represents a growing public health threat. Resistant bacteria can cause infections that are untreatable with most or all current antibiotics. For instance, carbapenem resistant enterobacteriaceae have been reported in nations including the United States, India, the UK, and others. ${ }^{1}$ Here, we develop a novel de novo aggregating antimicrobial peptide (AMP) and repurpose an RK2-mediated bacterial conjugation system to deliver the gene encoding this peptide. The toxicity of aggregating peptides arises from disruptive interaction of exposed hydrophobic side chains with cellular proteins, induction of oxidative stress, overload of proteolytic machinery, and co-aggregation with endogenous macromolecules. ${ }^{2}$ Instead of binding a particular macromolecular target site, aggregating AMPs cause widespread disruption of homeostasis in bacteria, potentially slowing resistance since many resistant phenotypes involve target site alterations. Bednarska et al. and Khodaparast et al. demonstrated the promise of aggregating AMPs $^{3,4}$ using peptides derived from existing bacterial protein sequences by predicting aggregation propensity with the statistical thermodynamics algorithm TANGO. ${ }^{5}$ A small fraction of the screened peptides showed significant antibacterial activity. However, the approaches were limited since the peptides were derived from naturally-occurring bacterial sequences and required screening of numerous candidates. Even the successful peptides were shown to lose function upon sequence rearrangements, indicating that they would not be amenable to directed evolution efforts which otherwise could restore activity if resistance was to arise.

The broad-host-range conjugative plasmid RK2 was originally isolated from antibiotic resistant Pseudomonas aeruginosa and Enterobacter aerogenes strains at the Birmingham Accident Hospital in $1969 .{ }^{6}$ Under optimal conditions, RK2, and its shuttle plasmids have very high conjugation frequencies. ${ }^{7-9}$ For instance, RK2 has been shown to mobilize shuttle plasmids 
from donor to recipient $E$. coli and from donor $E$. coli to recipient $P$. aeruginosa with conjugation frequencies of 8 and 0.2 transconjugants per donor (respectively). ${ }^{9}$ The RK2 plasmid can be efficiently mobilized among most gram-negative and many gram-positive bacteria. ${ }^{10}$ The pET11a backbone was chosen over RK2 itself so that its higher copy number of 15-20 copies per cell ${ }^{11}$ (with its pBR322 OriR) relative to RK2's 4-7 copies per cell ${ }^{12}$ and strong T7 promoter would maximize OpaL expression in targeted bacteria. This system might be particularly useful for treating infections which involve biofilms since the rate of bacterial conjugation greatly increases in biofilms, even up to 1,000 -fold. ${ }^{13-15}$ Precedent for such conjugative delivery can be found in studies that have used bacterial conjugation to deliver antibacterial CRISPR systems ${ }^{16-18}$ and toxic hyper-replicating plasmids..$^{19,20}$

We rationally design a de novo aggregating antimicrobial peptide, OpaL (Overexpressed protein aggregator Lipophilic), by choosing numerous hydrophobic amino acid residues to maximize protein aggregation (Fig. 1A). To the best of our knowledge, OpaL represents the first aggregating antimicrobial peptide designed without using preexisting sequences found in nature. We show that OpaL causes aggregation in bacteria and exhibits a bactericidal effect. We use the conjugative plasmid RK2 to transfer a pET11a-opaL shuttle plasmid from donor to recipient bacteria and target the strain of interest via a strain-specific promoter. Our work provides a new therapeutic strategy with potential clinical applications.

\section{Results and Discussion}

We rationally design a de novo aggregating antimicrobial peptide, OpaL (Overexpressed

protein aggregator Lipophilic), by choosing numerous hydrophobic amino acid residues to maximize protein aggregation (Fig. 1A). To the best of our knowledge, OpaL represents the first 
aggregating antimicrobial peptide designed without using preexisting sequences found in nature. We show that OpaL causes aggregation in bacteria and exhibits a bactericidal effect. We use the conjugative plasmid RK2 to transfer a pET11a-opaL shuttle plasmid from donor to recipient bacteria and target the strain of interest via a strain-specific promoter. Our work provides a new therapeutic strategy with potential clinical applications.

OpaL's sequence was manually constructed while considering design parameters around hydrophobic aggregation and intracellular half-life (Fig. 1A). To promote insolubility, 139 amino acids of OpaL's 185 residue sequence (75.1\%) possess hydrophobic side chains. These include 4 (2.2\%) alanine, $15(8.1 \%)$ cysteine, $53(28.6 \%)$ isoleucine, 17 (9.2\%) methionine, 14 (7.6\%) proline, and 36 (19.5\%) valine (Fig. 1B). As long stretches of hydrophobic residues further promote aggregation, ${ }^{21}$ most of OpaL consists of consecutive nonpolar amino acid sequences, though intermittent glycines were included since increased conformational flexibility has also been known to increase aggregate formation when hydrophobic amino acids are prevalent. ${ }^{22,23} \mathrm{OpaL}$ was made to be relatively large (with a molecular weight of about $18.5 \mathrm{kDa}$ ) compared to many antimicrobial peptides because amorphous aggregates are favored for larger proteins. ${ }^{24}$ Terminal polar amino acids (serine, asparagine, and threonine) were incorporated to avoid degradation by proteases which vastly shorten protein half-lives by recognizing bulky terminal hydrophobic residues. ${ }^{25} \mathrm{~N}$-formylmethionine was still allowed at the $\mathrm{N}$-terminus since, despite its hydrophobicity, N-formylmethionine promotes long protein half-lives. ${ }^{26}$ Since the $\mathrm{N}$ formylmethionine found in bacterial proteins is often cleaved by proteases, the next several chosen residues were also among those which promote long half-lives. OpaL's hydrophobicity-centered design leaves it amenable to a rich array of sequence variations since a large number of possible rearrangements would still retain OpaL's extremely hydrophobic character and therefore opens the 
door to the development of a new class of aggregating antimicrobial peptide.

We also created a similar 184 residue control peptide called OpaLacidic which possesses markedly less potential for hydrophobic aggregation. OpaLacidic includes 30 aspartic acid residues comprising $16.3 \%$ of the sequence (supplementary Fig. S1). To facilitate charge-charge repulsion and interfere with hydrophobic aggregation, these aspartic acids were placed every five residues over most of the sequence. This peptide served to highlight the importance of OpaL's hydrophobic aggregation mechanism.

We demonstrated OpaL's intracellular toxicity by expressing it from the strong T7 promoter on the pET11a-opaL shuttle plasmid in E. coli BL21 (DE3). In pET11a-derived vectors, a lac operator is located downstream of the T7 promoter, allowing induction with isopropyl $\beta$-D1-thiogalactopyranoside (IPTG) (Fig. 1A). When measuring the optical density (OD) of bacterial cultures of BL21 (DE3) carrying pET11a-opaL, OpaL expression with $1.0 \mathrm{mM}$ IPTG completely precluded growth (Fig. 2A). With 0.1 mM IPTG, OpaL expression decreased mean OD (Fig. 2B), but some growth occurred. Without IPTG, BL21 (DE3) carrying pET11a-opaL showed a longer lag time compared to BL21 (DE3) not carrying any plasmids, potentially due to leaky expression from the opaL gene (Fig. 2C). BL21 (DE3) expressing OpaLacidic displayed significantly lower toxicity than OpaL, albeit with lengthened lag times relative to control bacteria not carrying any plasmids (Fig. 2A-C). To further investigate this, we deleted the open reading frame of opaL to

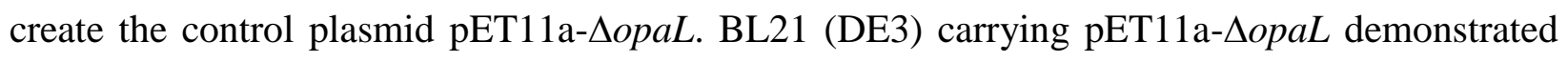
significantly higher growth than cells expressing OpaL or OpaLacidic, although upon induction (0.1 and 1.0 mM IPTG) a lower growth plateau and longer lag time were observed compared to the control without any plasmid (Fig. 2A-B) possibly due to metabolic burden from the transcription of short RNAs containing any remaining sequence between the promoter and 
terminator that was not deleted.

In colony forming unit (CFU) experiments, viable cell counts of BL21 (DE3) expressing OpaL dropped to zero after 4 hours of induction with $1.0 \mathrm{mM} \mathrm{IPTG}(\mathrm{p}<0.01)$, indicating a bactericidal mechanism of action (Fig. 2D). Without IPTG, the viable cell count continued to increase significantly over time. Viable cell counts after 2 hours and 4 hours for BL21 (DE3) carrying pET11a-opaLacidic remained relatively constant ( $\mathrm{p}>0.05$ ) with $1.0 \mathrm{mM}$ IPTG indicating a weaker bacteriostatic effect compared to OpaL (Fig. 2E). Without IPTG, bacterial CFU increased over time. These results confirm the antibacterial effect of OpaL.

To computationally test OpaL's aggregation, we employed the statistical thermodynamics algorithm TANGO. ${ }^{5}$ OpaL showed an extremely high mean aggregation propensity of $35.7 \%$ (values greater than $5 \%$ predict aggregation). ${ }^{5}$ OpaLacidic showed a low mean aggregation propensity of $0.7 \%$ (Fig. 3A). These data support OpaL's formation of hydrophobic aggregates. We also used QUARK, an ab initio protein structure prediction tool, to predict the tertiary structures of OpaL and OpaLacidic. DeepView was employed to visualize these structures and compute their solvent-exposed hydrophobic surface areas. OpaL was predicted to be rich in $\beta$ sheet structures (Fig. 3B), while OpaLacidic was mostly composed of unstructured loops (Fig. 3C). OpaL's greater antibacterial toxicity relative to OpaLacidic is consistent with these results since many pathological protein aggregates are also rich in $\beta$-sheets. ${ }^{5,27}$ OpaL's predicted structure demonstrated $43.6 \%$ hydrophobic surface area, while OpaLacidic demonstrated a predicted hydrophobic surface area of $21.2 \%$ (Fig. 3B-C). Again, OpaL's higher antibacterial toxicity relative to OpaLacidic is consistent with these results since protein aggregation is known to be heavily dependent on hydrophobicity. ${ }^{2,5,28}$

To provide experimental evidence for OpaL's aggregation, we performed an aggregation 
assay by staining host BL21 (DE3) with the dye nile red which fluoresces upon exposure to hydrophobic environments. ${ }^{29}$ After induction with $1.0 \mathrm{mM}$ IPTG, cells carrying pET11a-opaL showed significantly greater increases in fluorescence relative to cells with pET11a-opaLacidic, pET11a- $\triangle o p a L$, or cells without any plasmids $(\mathrm{p}<0.01)$ (Fig. 3D). After induction with $0.1 \mathrm{mM}$ IPTG, cells expressing OpaL showed higher increases in fluorescence relative to the other groups, though the differences were not statistically significant. These results are consistent with the data from TANGO and QUARK, further supporting OpaL's mechanism of hydrophobic aggregation. We also extracted total and soluble protein fractions from strains expressing OpaL, OpaLacidic and $\Delta \mathrm{OpaL}$ (supplementary Fig. S3A-B). The insoluble protein fraction showed substantially more bands (and higher intensity bands) only with expression of OpaL (supplementary Fig. S3A), while the soluble fraction remained similar for OpaL, OpaLacidic and $\Delta \mathrm{OpaL}$ strains (supplementary Fig. S3B). Finally, we used laser scanning confocal fluorescence microscopy to directly visualize intracellular OpaL aggregates stained with nile red (Fig. 3E). Bacteria expressing OpaL exhibited visible inclusion body formation, while aggregates were not observed in controls with OpaLacidic and $\Delta \mathrm{OpaL}$.

We show that bacterial conjugation facilitates delivery of opaL-carrying donor cells to targeted BL21 (DE3) recipient cells that express the T7 RNA polymerase (Fig. 4A). To mobilize opaL, we chose the RK2 plasmid for its high transfer frequency, conjugative promiscuity, and stability. ${ }^{6,10}$ To deliver opaL into recipient cells, we designed the pET11a-opaL shuttle plasmid which includes a 450 bp sequence identical to the origin of transfer (OriT) site in RK2 $2^{30}$ and a chloramphenicol $(\mathrm{Cm})$ resistance gene (supplementary Fig. S2). We co-transformed both RK2 and pET11a-opaL into E. coli C600 to create donor cells. Since E. coli C600 do not express T7 polymerase, OpaL expression does not occur in host donor cells. Future extensions of this may 
allow promoter-based targeting of pathogenic microorganisms via pathogen-specific expression of T7 RNA polymerase. We confirmed that, when mobilized by RK2, pET11a-opaL is transferred conjugatively by measuring mating frequency between donor and recipient cells $\left(2.63 \times 10^{-}\right.$ ${ }^{2} \pm 1.23 \times 10^{-2}$ transconjugants per recipient).

We mated donor E. coli C600 carrying RK2 and pET11a-opaL plasmids with recipient BL21 (DE3) carrying the GFP-expressing pHL662 plasmid using OD-adjusted 1:3 donor to recipient ratios and 1.0 mM IPTG. GFP fluorescence was employed to distinguish between strains (supplementary Fig. S4A). Ratios of recipient colonies to total colonies were computed. The experimental group's recipient CFU fractions were 2.0 -fold lower than the fractions of the mating control in which donors only carried RK2 (p<0.01) (Fig. 4B). We also measured recipient growth curves using GFP fluorescence from mating cultures in a microplate reader. Recipient BL21 (DE3) demonstrated significantly lower growth when donors delivered both RK2 and pET11a-opaL compared to the control (Fig. 4C) $(\mathrm{p}<0.001)$.

We performed three-strain matings between donor C600 carrying RK2 and pET11a-opaL plasmids, targeted recipient BL21 (DE3) with pHL662, and non-target recipient E. coli DH5 $\alpha$ expressing mCherry from the pUV145 plasmid $^{31}$ (supplementary Fig. S4B) using a 1:1:2 ODadjusted ratio of targeted recipients to non-target recipients to donors. The ratio was chosen because it gives an approximately equal number of donor cells relative to total recipient cells. Fractions of targeted recipients and fractions of non-target recipients were determined relative to total colonies on each plate. The experimental group's targeted recipient CFU fractions were 2.7fold lower than those in the mating control $(\mathrm{p}<0.001)$, while the non-target recipient $\mathrm{CFU}$ fractions were not significantly different from the control ( $\mathrm{p}=0.71$ ) (Fig. 4D).

We designed and tested OpaL as a basis for rational design of novel antimicrobials. 
Aggregating peptides offer a new approach to addressing bacterial resistance, since unlike smallmolecule antibiotics, hydrophobic aggregates disrupt homeostasis rather than binding to a specific macromolecular target site. ${ }^{2,28}$ Furthermore, insoluble aggregates may show less susceptibility to efflux and OpaL's de novo character may decrease the frequency of enzymatic exaptation towards specific binding and cleavage of sequence motifs. Even if resistance was to arise, OpaL's design

is highly amenable to directed evolution. Because OpaL's aggregation centrally depends on its hydrophobic characteristics, mutations should be less likely to decrease OpaL's activity, widening the pool of potentially improved mutants as compared to most protein therapeutics. ${ }^{32}$ After iterated mutagenesis, $o p a L$ may regain activity against resistant pathogens. Mutations in $o p a L$ 's promoter may enable outgrowth, but donors with fresh copies of the original opaL could be introduced to restore full expression. Given that hydrophobic aggregation can occur in any aqueous cellular environment, OpaL may exhibit activity in diverse types of bacteria. This technology provides new opportunities for addressing antibiotic-resistant infections.

\section{Methods}

Strains, plasmids, kits, and gene synthesis

The pET11a-opaL and pET11a-opaLacidic vector designs were constructed by GenScript using their artificial gene synthesis and custom cloning services. The opaL open reading frame, opaLacidic open reading frame, RK2 OriT, and chloramphenicol resistance $(\mathrm{CmR})$ gene were artificially synthesized. RK2 was obtained in E. coli $\mathrm{C} 600\left(\mathrm{ATCC}^{\circledR} 37125^{\mathrm{TM}}\right)$. We used a Mix \& Go E. coli Transformation Kit from Zymo Research to induce chemical competence in the E. coli C600 (RK2) before transforming with pET11a-opaL. To prevent loss of RK2, we grew these cells under kanamycin selection. E. coli C600 (RK2, pET11a-opaL) and E. coli C600 (RK2) were 
subsequently used as donor bacteria.

The pHL662 plasmid, carried by E. coli XL1 Blue, was Addgene vector $37636 .{ }^{33}$ The $E$. coli XL1 Blue was used as recipient bacteria for measuring the mating frequency. We isolated pHL662 using a Zymo Research Zyppy Plasmid Miniprep Kit. Chemically competent E. coli BL21 (DE3) were acquired from NEB and transformed with pHL662. These E. coli BL21 (DE3) (pHL662) were used as recipients in the mating-toxicity assays. Separate samples of E. coli BL21 (DE3) were also transformed with pET11a-opaL for toxicity assays. E. coli NEB10- $\beta$ was acquired from NEB and used as a host for propagating the pET11a- $\Delta o p a L$ vector. The pUV145 plasmid, ${ }^{31}$ was carried in an E. coli DH5 $\alpha$ host. E. coli DH5 $\alpha$ carrying pUV145 were employed in our three strain mating-toxicity assay. A list of all strains and plasmids used for this work is presented in supplementary table S1.

\section{Culture conditions}

Growth media included Luria Bertani (LB) broth (liquid medium) and LB agar (solid medium) with selective antibiotics as described for each experiment. Liquid cultures were incubated at $37^{\circ} \mathrm{C}$ in an orbital shaker or in a Tecan GENios plate reader. When using the plate reader, cultures were set to shake for 10 minutes, stand idle for 10 minutes, and then shake for an additional 10 seconds prior to taking a measurement. Solid cultures were grown in a stationary incubator at $37^{\circ} \mathrm{C}$. The opaL gene and the opaLacidic gene were induced using IPTG at concentrations of 1.0 or $0.1 \mathrm{mM}$ as described for each experiment. GFP from pHL662 and mCherry from pUV145 were induced using IPTG at concentrations of $1.0 \mathrm{mM}$. Except where otherwise noted, ampicillin was used to maintain pET11a-opaL and pET11a-opaLacidic and kanamycin was used to maintain RK2, pHL662, and pUV145.

\section{Molecular cloning}


The pET11a- $\triangle o p a L$ control plasmid was prepared by removing opaL's open reading frame (positions 6124 to 6690). To accomplish this, pET11a-opaL was first propagated in E. coli DH5 $\alpha$ to facilitate DNA methylation. Primers were designed to amplify the part of pET11a-opaL which excludes the opaL-containing sequence between pET11a-opaL plasmid's NdeI and BamHI cut sites. The forward primer (5'-ggaaggggatccggctgctaacaaag-3') still retained the original BamHI cut site sequence, while the reverse primer (5'-gaggagggatcctatatctccttcttaaagttaaacaaaat-3') included a small overhang which replaced the NdeI cut site with another BamHI cut site upon amplification. After amplifying this sequence, the PCR product was double digested with DpnI (a methylationdependent restriction enzyme) and BamHI. The purpose of using DpnI was to degrade any remaining background DNA which still contained opaL. Next, the linear vector was ligated overnight and then electroporated into E. coli NEB10- $\beta$. A transformant was picked and grown in liquid media overnight. The pET11a- $\triangle o p a L$ plasmid was miniprepped from this culture and then confirmed to have the correct size by performing gel electrophoresis alongside a sample of pET11a-opaL. Maps of plasmids used for this work are presented in supplementary Fig. S1.

\section{Toxicity assay using growth curves}

Overnight cultures (three biological replicates) of E. coli BL21 (DE3) carrying pET11aopaL, E. coli BL21 (DE3) carrying pET11a-opaLacidic, E. coli BL21 (DE3) carrying pET11a$\triangle o p a L$, and E. coli BL21 (DE3) without any plasmids were diluted $1 \times 10^{-4}$ and incubated for 2 hours. Samples from each culture were diluted 1:50 into fresh media with 1.0 mM IPTG, 0.1 mM IPTG, and 0.0 mM IPTG in a 96 well plate. Absorbance values were measured every 20 minutes for $20 \mathrm{~h}$ using the Tecan Genios plate reader settings described earlier. These data were normalized by subtracting the absorbance of the media and dividing by the OD at $\mathrm{t}=0$ for each sample.

Toxicity assay using Colony Forming Units (CFUs) 
We tested opaL's antibacterial activity in E. coli BL21 (DE3) carrying pET11a-opaL and E. coli BL21 (DE3) carrying pET11a-opaLacidic. Overnight starter cultures (three biological replicates) were diluted 1:1000 and incubated for 2 hours. Serial dilutions of these exponential cultures were plated on solid medium to obtain CFUs at $t=0 \mathrm{~h}$. The cultures were further diluted 1:100. Immediately after these dilutions, we split the cultures into control and experimental tubes and then added IPTG to the experimental tubes $(1.0 \mathrm{mM}$ final concentration) in order to induce opaL expression. Serial dilutions were then plated on solid media at $\mathrm{t}=2 \mathrm{~h}$ and $\mathrm{t}=4 \mathrm{~h}$.

\section{Computational prediction of aggregation using TANGO}

The online TANGO platform (http://tango.crg.es/protected/academic/calculation.jsp) was used to predict percent aggregation for OpaL and OpaLacidic. The same parameters were used for both peptides. We assumed OpaL and OpaLacidic concentrations of $2 \mathrm{mM}$ based on T7's known expression levels. ${ }^{34}$ Using standard physiological parameters for E. coli, ${ }^{35,36}$ we entered an ionic strength of $0.25 \mathrm{mM}$, a cytosolic $\mathrm{pH}$ of 7.5 , and a temperature of $37^{\circ} \mathrm{C}$. The $\mathrm{N}$ - and C-termini were given the default parameter of not having any chemical modifications.

\section{Computational structure prediction using QUARK}

The online QUARK platform ${ }^{37}$ (https://zhanglab.ccmb.med.umich.edu/QUARK/) was used to predict tertiary structures for OpaL and OpaLacidic. The raw amino acid sequences were inputted into the algorithm and the results retrieved in PDB file format. From these files, 3D graphics were created with DeepView v4.1.0. ${ }^{38}$ DeepView was also used to compute OpaL and OpaLacidic's solvent-exposed hydrophobic surface areas.

Nile red aggregation assay

Overnight cultures (ten biological replicates) of E. coli BL21 (DE3) carrying pET11aopaL, E. coli BL21 (DE3) carrying pET11a-opaLacidic, E. coli BL21 (DE3) carrying pET11a- 
$\triangle o p a L$, and E. coli BL21 (DE3) without any plasmids were diluted 1:10 into $900 \mu \mathrm{L}$ of fresh media and incubated for $1 \mathrm{~h}$ before being pelleted, washed, and resuspended in Phosphate Buffered Saline (PBS). Nile red was added to a final concentration of $1.0 \mu \mathrm{g} / \mathrm{mL}$. Absorbance was measured at 590 $\mathrm{nm}$ and fluorescence was measured at $590 \mathrm{~nm}$ excitation and $610 \mathrm{~nm}$ emission in a Tecan Genios plate reader. Next, IPTG was added to five of the replicates to a concentration of $1.0 \mathrm{mM}$ and the other five replicates to a concentration of $0.1 \mathrm{mM}$. These samples were then incubated at $37^{\circ} \mathrm{C}$ for $2 \mathrm{~h}$ with shaking. Absorbance and fluorescence were measured again at the same wavelengths. Initial and final fluorescence values were normalized to the corresponding absorbance values and the overall normalized changes in fluorescence were calculated.

\section{Fluorescence microscopy to visualize aggregates}

Pairs of overnight cultures of E. coli BL21 (DE3) carrying pET11a-opaL, E. coli BL21 (DE3) carrying pET11a-opaLacidic, and E. coli BL21 (DE3) carrying pET11a-4opaL were diluted 1:10 and incubated for 90 minutes with shaking at $37^{\circ} \mathrm{C}$. IPTG was then added to half of the cultures at final concentrations of $1.0 \mathrm{mM}$ and all the cultures were incubated for another 90 minutes. Next, the cells were pelleted, washed, and resuspended in PBS with 4\% formaldehyde. The bacteria and intracellular aggregates were imaged using a Nikon A1R laser scanning confocal microscope with a 100x objective lens (numerical aperture 1.4), a $561 \mathrm{~nm}$ laser, and a 595/50 nm emission filter.

\section{Protein gels}

Overnight cultures of E. coli BL21 (DE3) carrying pET11a-opaL, E. coli BL21 (DE3) carrying pET11a-opaLacidic, and E. coli BL21 (DE3) carrying pET11a-AopaL were diluted 1:10 into $5 \mathrm{~mL}$ of fresh media and incubated for 90 minutes with shaking at $37^{\circ} \mathrm{C}$. IPTG was then added to each culture at final concentrations of $1.0 \mathrm{mM}$ and the cultures were incubated for another 90 
minutes. Next, the cells were pelleted, washed, and resuspended in PBS. The samples were sonicated for 30 seconds each. Total protein fractions and soluble protein fractions were run on an $8 \%$ Tris-Glycine polyacrylamide gel. The total fractions were taken directly from the lysed samples while the soluble fractions were taken from the supernatant after pelleting the total fractions. The gel was run at $4^{\circ} \mathrm{C}$ for 1.5 hours using $150 \mathrm{~V}, 160 \mathrm{~mA}$ conditions. The gel was then fixed and stained using Coomassie Brilliant Blue R-250 dye. The gel was imaged using a Bio-Rad Gel Doc ${ }^{\mathrm{TM}}$ EZ Gel Imager.

\section{Mating frequency assay}

Mating frequency assays were performed to confirm that RK2 and pET11a-opaL are capable of conjugative transfer. $1 \mathrm{~mL}$ overnight cultures (three biological replicates) of E. coli C600 donors carrying both RK2 and pET11a-opaL, E. coli C600 donors carrying the only RK2, and E. coli XL1 Blue recipients carrying pHL662 were pelleted and washed to remove antibiotics before being resuspended in $250 \mu \mathrm{L}$ of media. The volumes of these cultures were adjusted to have equal OD values before mating cultures with 1:5 donor to recipient ratios were made by volume. E. coli C600 (RK2, pET11a-opaL) and E. coli C600 (RK2) were each separately paired with the recipients. $20 \mu \mathrm{L}$ of the mating cultures were spotted on LB agar plates without selection and incubated for 5 hours. Next, we cut out solid agar slices with the spots and transferred them to liquid cultures without selection. After $1 \mathrm{~h}$ of incubation, the cultures were diluted 1:10,000 and plated on X-Gal with appropriate antibiotics.

X-Gal allowed distinction between blue donor (E. coli C600) colonies and white transconjugant (E. coli XL1 Blue) colonies. E. coli XL1 Blue possess the $\Delta$ lacZ genotype and so cannot metabolize X-Gal to produce blue pigment. Donors and transconjugants with both RK2 and pET11a-opaL were selected with chloramphenicol while donors and transconjugants with only 
RK2 were selected with ampicillin. Control X-Gal plates without antibiotics were made for each mating culture. Mating frequencies were determined by taking the ratio of transconjugant colonies to total recipient colonies.

Two strain mating-toxicity assay using CFUs

Mating-toxicity assays using CFUs demonstrated the functionality of our bacterial conjugation delivery system for transferring opaL to target bacteria. $1 \mathrm{~mL}$ overnight cultures were made with three biological replicates of E. coli C600 donors carrying both RK2 and pET11a-opaL, E. coli C600 donors carrying only RK2, and E. coli BL21 (DE3) recipients carrying pHL662. These cultures were then diluted 1:100 in $5 \mathrm{~mL} \mathrm{LB}$ medium and incubated for $3 \mathrm{~h}$, followed by pelleting and washing twice with $\mathrm{LB}$ to remove antibiotics, and then resuspension in $250 \mu \mathrm{L}$ of fresh medium. Culture volumes were adjusted to give approximately equivalent OD values. Next, donor and recipient strains were mixed to create mating cultures with 1:3 donor to recipient ratios. Mating cultures were spotted onto $1.0 \mathrm{mM}$ IPTG plates without selection and incubated for $5 \mathrm{~h}$. We cut out the solid agar slices with mating spots, transferred them each into $1 \mathrm{~mL}$ of PBS, and vortexed thoroughly to resuspend the mated bacteria. These cells were diluted to $1 \times 10^{-5}$ and plated on LB agar plates containing kanamycin (to maintain pHL662) and 1.0 mM IPTG. GFP-expressing (recipient) colonies and non-fluorescent (donor) colonies were counted using $470 \mathrm{~nm}$ excitation and $530 \mathrm{~nm}$ emission wavelengths.

Two strain mating-toxicity assay using fluorescence growth curves

Mating-toxicity assays using fluorescence growth curves further showed the functionality of our bacterial conjugation delivery system for the opaL gene. $1 \mathrm{~mL}$ overnight cultures were made with four biological replicates of $E$. coli C600 donors carrying both RK2 and pET11a-opaL, E. coli C600 donors carrying only RK2, and E. coli BL21 (DE3) recipients carrying pHL662. The 
overnight cultures were washed twice to remove antibiotics and resuspended in $250 \mu \mathrm{L}$ of LB media. Culture volumes were adjusted to give approximately equivalent OD values. Next, donor and recipient strains were mixed to create mating cultures with 1:1 donor to recipient ratios. Mating cultures were spotted onto $1.0 \mathrm{mM}$ IPTG plates without selection and incubated for $5 \mathrm{~h}$. We cut out the solid agar slices with mating spots and transferred them to liquid media, where they were incubated for $1 \mathrm{~h}$. The cultures were diluted 1:20 into fresh media with 1.0 mM IPTG in a 96 well plate. GFP fluorescence was measured with $485 \mathrm{~nm}$ excitation and $535 \mathrm{~nm}$ emission every 20 minutes for $20 \mathrm{~h}$ using the Tecan Genios plate reader settings described earlier. These data were normalized with the media's autofluorescence values.

\section{Three strain mating-toxicity assay using CFUs}

We performed a three strain mating-toxicity assay to demonstrate that this conjugation based delivery approach functions effectively when bacteria other than the donors and the targeted recipients are present. $1 \mathrm{~mL}$ overnight cultures were made with three biological replicates of $E$. coli C600 donors carrying both RK2 and pET11a-opaL, E. coli C600 donors carrying only RK2, E. coli BL21 (DE3) recipients carrying pHL662, and E. coli DH5 $\alpha$ recipients carrying pUV145. As in the two strain assay, the overnight cultures were diluted 1:100 in $5 \mathrm{~mL} \mathrm{LB}$ and incubated for $3 \mathrm{~h}$, followed by washing twice to remove antibiotics, and resuspension in $250 \mu \mathrm{L}$ of media. Culture volumes were adjusted to give approximately equivalent OD values. Recipient E. coli BL21 (DE3), recipient E. coli DH5 $\alpha$, and donor E. coli C600 were mixed to create mating cultures with ratios of 1:1:2 respectively. Mating cultures were spotted onto $1.0 \mathrm{mM}$ IPTG plates without selection and incubated for 5 hours. Equivalent volumes of E. coli DH5 $\alpha$ alone were spotted onto $1.0 \mathrm{mM}$ IPTG plates without selection and incubated for $5 \mathrm{~h}$. We cut out the solid agar slices with mating spots, transferred them each into $1 \mathrm{~mL}$ of PBS, and vortexed thoroughly to resuspend the 
mated bacteria. These cells were diluted to $1 \times 10^{-5}$ and plated on kanamycin (to maintain pHL662 and pUV145) and 1.0 mM IPTG. GFP-expressing (target recipient) colonies and non-fluorescent (donor) colonies were counted using $470 \mathrm{~nm}$ excitation and $530 \mathrm{~nm}$ emission wavelengths, while mCherry-expressing (non-target recipient) colonies were counted using $540 \mathrm{~nm}$ excitation and 590 nm emission wavelengths.

\section{Figures and tables}

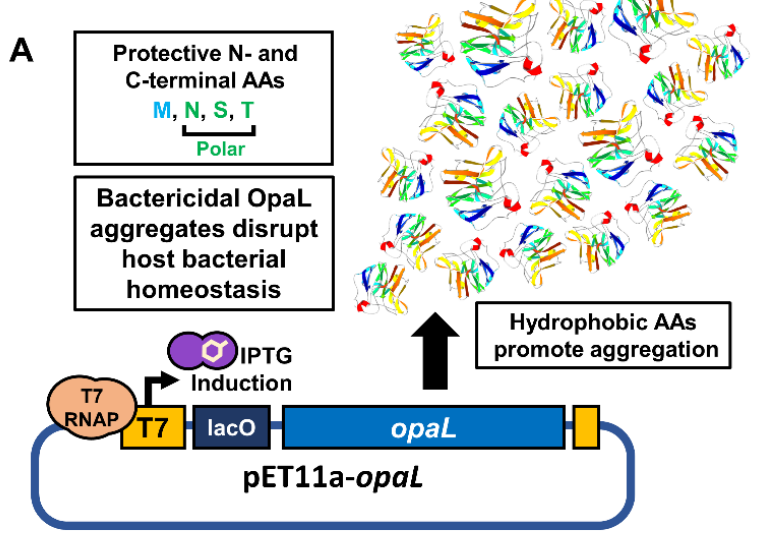

B

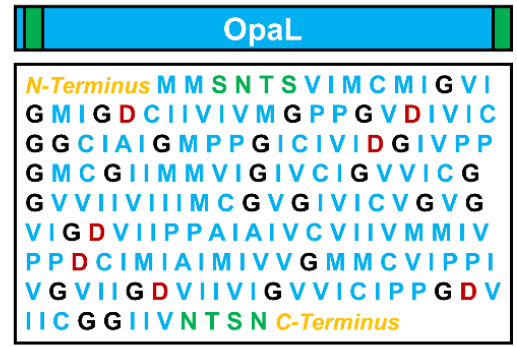

$75.1 \%$ Hydrophobic $3.8 \%$ Acidic

Figure 1. Rational design of OpaL. (A) The pET11a-opaL vector expresses OpaL from the T7 promoter and so restricts expression to the target strain, BL21 (DE3). Polar terminal patches were incorporated in OpaL to increase OpaL's intracellular half-life. ${ }^{25}$ Numerous $(75.1 \%)$ hydrophobic residues facilitate formation of toxic intracellular aggregates. ${ }^{2,28}$ (B) OpaL's primary amino acid sequence with hydrophobic amino acids in blue, glycines in black, aspartic acids in red, and polar amino acids in green. 

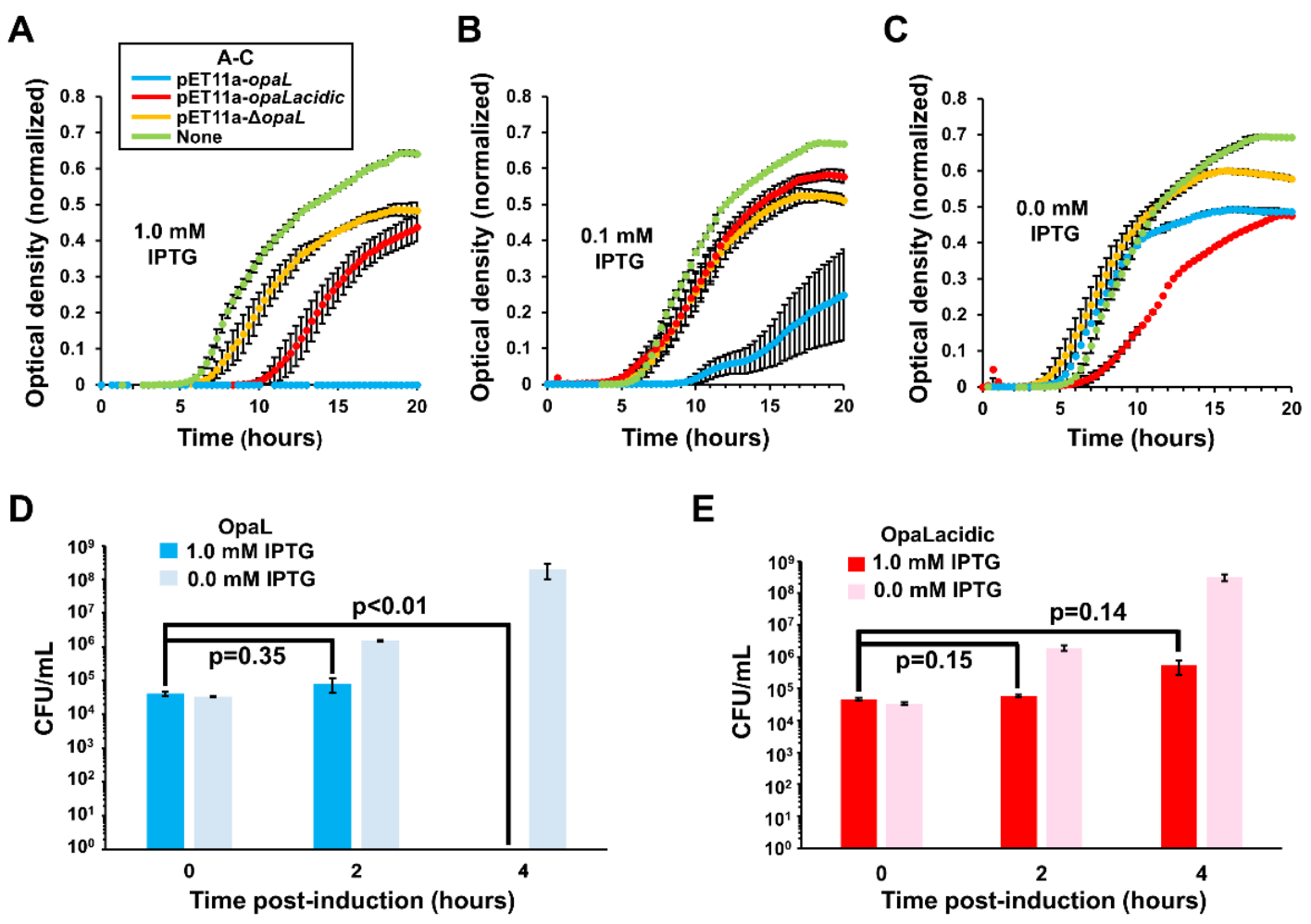

Figure 2. Antimicrobial effect of OpaL. (A-C) Growth curves of BL21 (DE3) carrying plasmids as labeled under (A) $1.0 \mathrm{mM}$ IPTG induction, (B) $0.1 \mathrm{mM}$ IPTG induction, and (C) without IPTG induction. (D) CFUs of BL21 (DE3) carrying pET11a-opaL at $\mathrm{t}=0$, $\mathrm{t}=2$, and $\mathrm{t}=4$ hours post induction with 1.0 mM IPTG and at the same time points without IPTG. (E) CFUs of BL21 (DE3) carrying pET11a-opaLacidic at $\mathrm{t}=0, \mathrm{t}=2$, and $\mathrm{t}=4$ hours post induction with $1.0 \mathrm{mM}$ IPTG and without IPTG. These data represent the means of three biological replicates. Error bars represent standard error and $P$-values were calculated using a two-tailed type II t-test. 

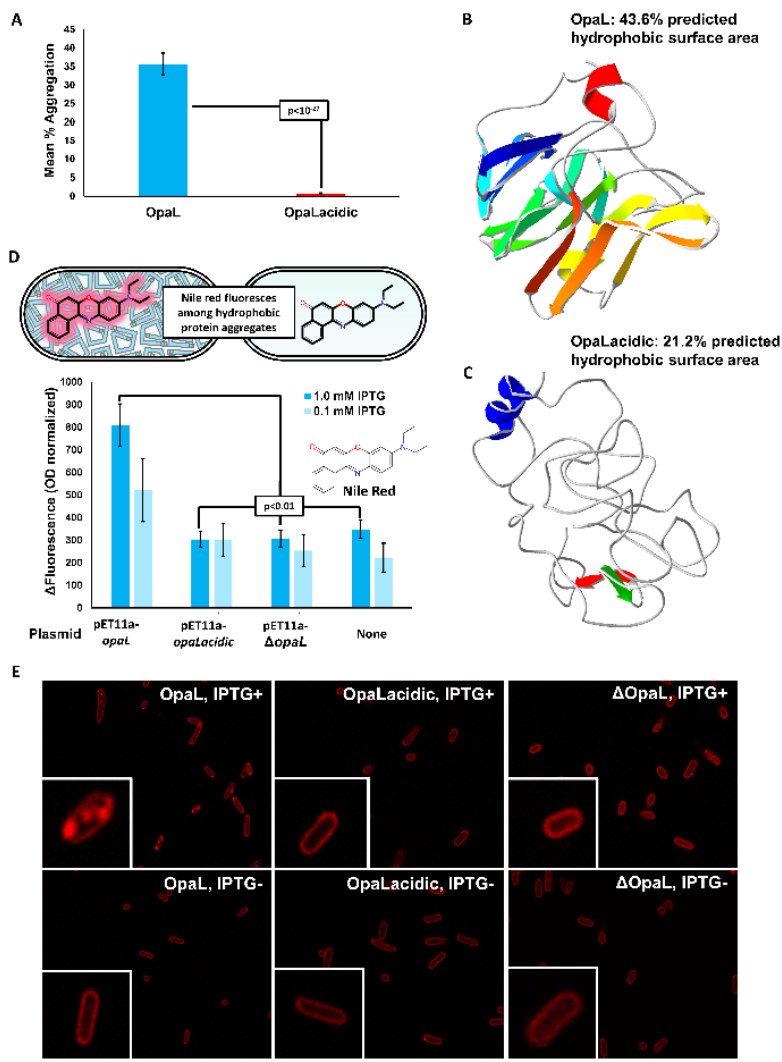

Figure 3. OpaL causes intracellular aggregate formation. (A) Predicted mean aggregation propensity percentages for OpaL and OpaLacidic using the TANGO algorithm. (B) OpaL's structure as predicted by the QUARK algorithm. (C) The structure of OpaLacidic as predicted by QUARK. (D) BL21 (DE3) carrying plasmids as labeled were stained with nile red to show that OpaL forms hydrophobic aggregates. These data represent the means of five biological replicates. Error bars represent standard error and $P$-values were calculated using a two-tailed type II t-test. (E) Intracellular OpaL aggregates visualized with fluorescence microscopy and nile red. Aggregates are visible in the bacteria expressing OpaL (top leftmost panel), but not in the controls. 

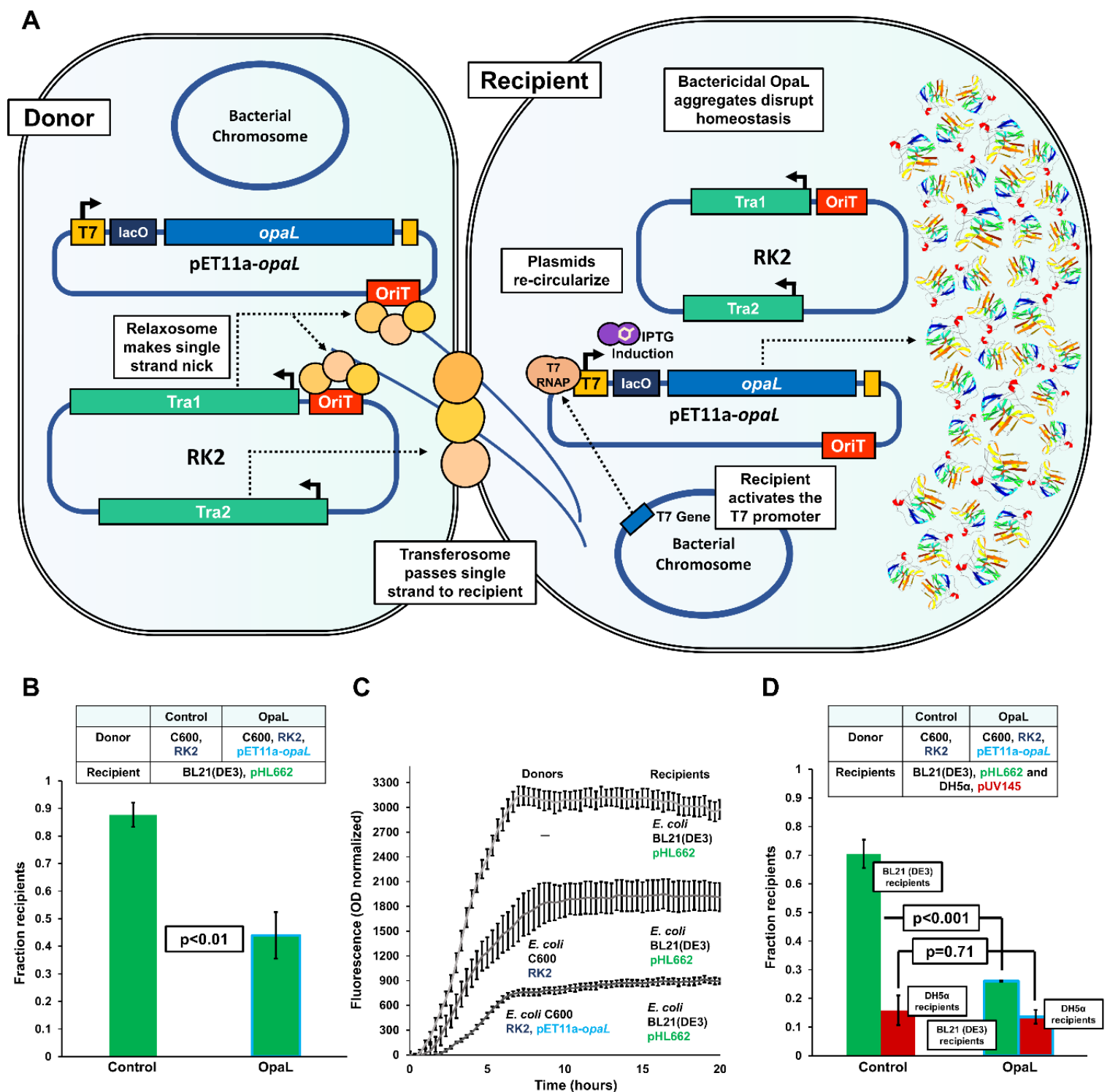

Figure 4. OpaL shows targeted killing when delivered by bacterial conjugation. (A) Donor

bacteria transfer the broad-host-range conjugative plasmid RK2 and the shuttle plasmid pET11aopaL to recipient bacteria. RK2 encodes a relaxosome complex which makes a single-stranded nick in the origin of transfer (OriT) ${ }^{39}$ and a transferosome complex which facilitates the transfer of the ssDNA to the recipient. The $450 \mathrm{bp}$ OriT sequence from RK2 was cloned into pET11a-opaL to facilitate conjugative transfer of opaL. BL21 (DE3) encodes T7 RNA polymerase which binds the T7 promoter upstream of $o p a L$ and initiates expression, allowing OpaL to kill the targeted host 
bacterium. (B) Two-strain CFU mating-toxicity assay as measured by the ratio of BL21 (DE3) recipient $\mathrm{CFU} / \mathrm{mL}$ to total $\mathrm{CFU} / \mathrm{mL}$. (C) Two-strain growth curve mating-toxicity assay measured via recipient GFP fluorescence normalized to OD. These data represent the means of four biological replicates and error bars represent standard error. (D) Three-strain CFU mating-toxicity assay as measured by the ratios of BL21 (DE3) recipient CFU/mL to total CFU/mL and DH5 $\alpha$ non-target recipient $\mathrm{CFU} / \mathrm{mL}$ to total $\mathrm{CFU} / \mathrm{mL}$. For panels $\mathrm{B}$ and $\mathrm{D}$ the mean recipient fractions relative to total cells are displayed, the data represent the means of three biological replicates, error bars represent standard error, and $P$-values were calculated using a two-tailed type II t-test.

\section{OpaLacidic}

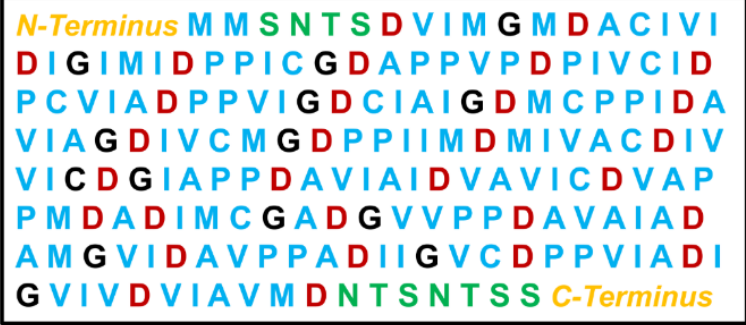

$70.7 \%$ Hydrophobic $16.3 \%$ Acidic

Supplementary Figure S1. The amino acid sequence of OpaLacidic. Unlike OpaL, the OpaLacidic peptide's hydrophobic patches are much more frequently interrupted by aspartic acid residues. In addition, OpaLacidic has a higher proline content, a longer stretch of C-terminal polar residues, and alternatively ordered residues within its hydrophobic patches. 

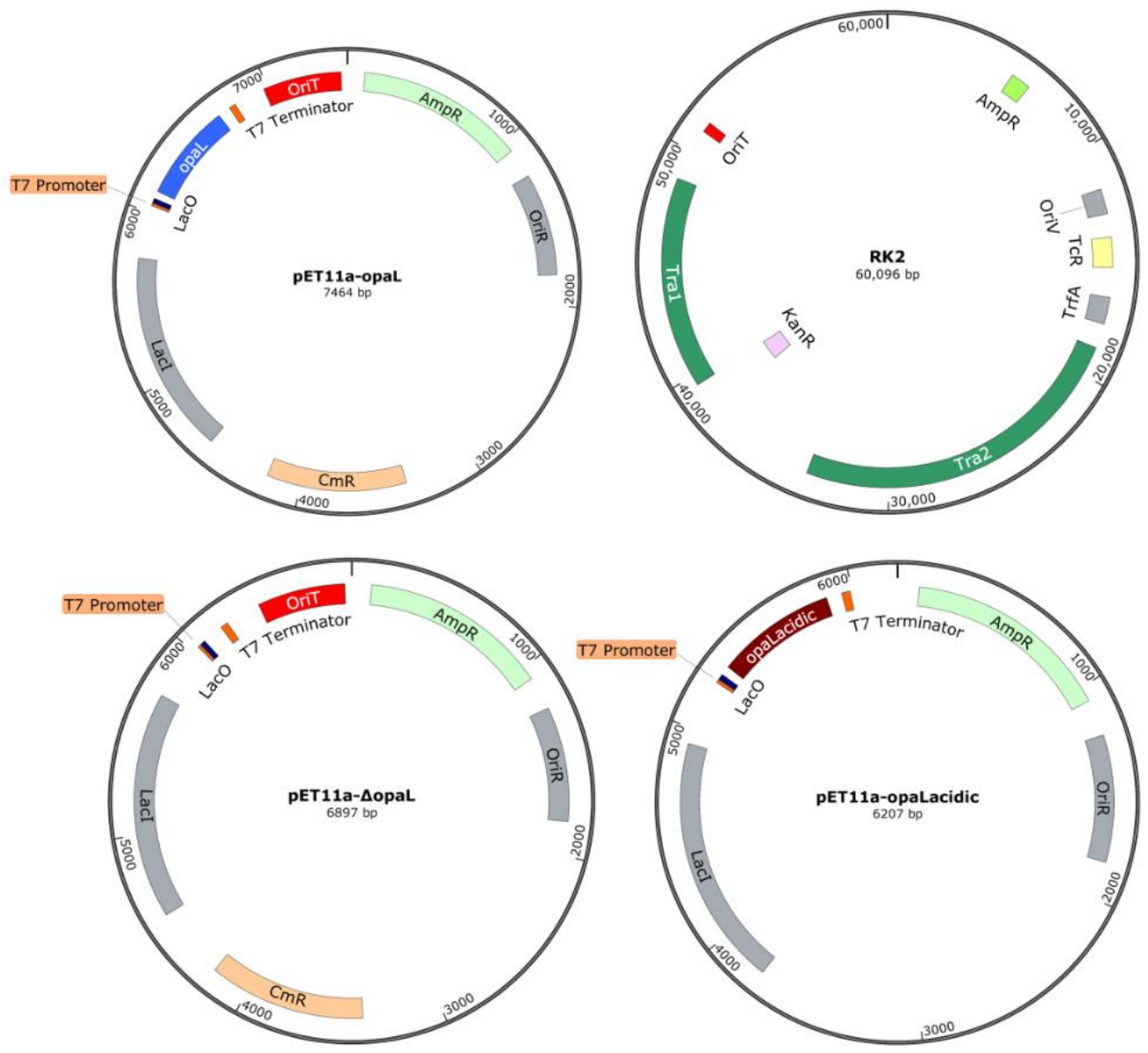

Supplementary Figure S2. Plasmid maps for the broad-host range conjugative plasmid RK2, the shuttle vector pET11a-opaL which includes an RK2 OriT sequence and the opaL gene, the pET11a-opaLacidic plasmid, and the control vector pET11a-_opaL which does not have an open reading frame ahead of the $\mathrm{T} 7$ promoter. 

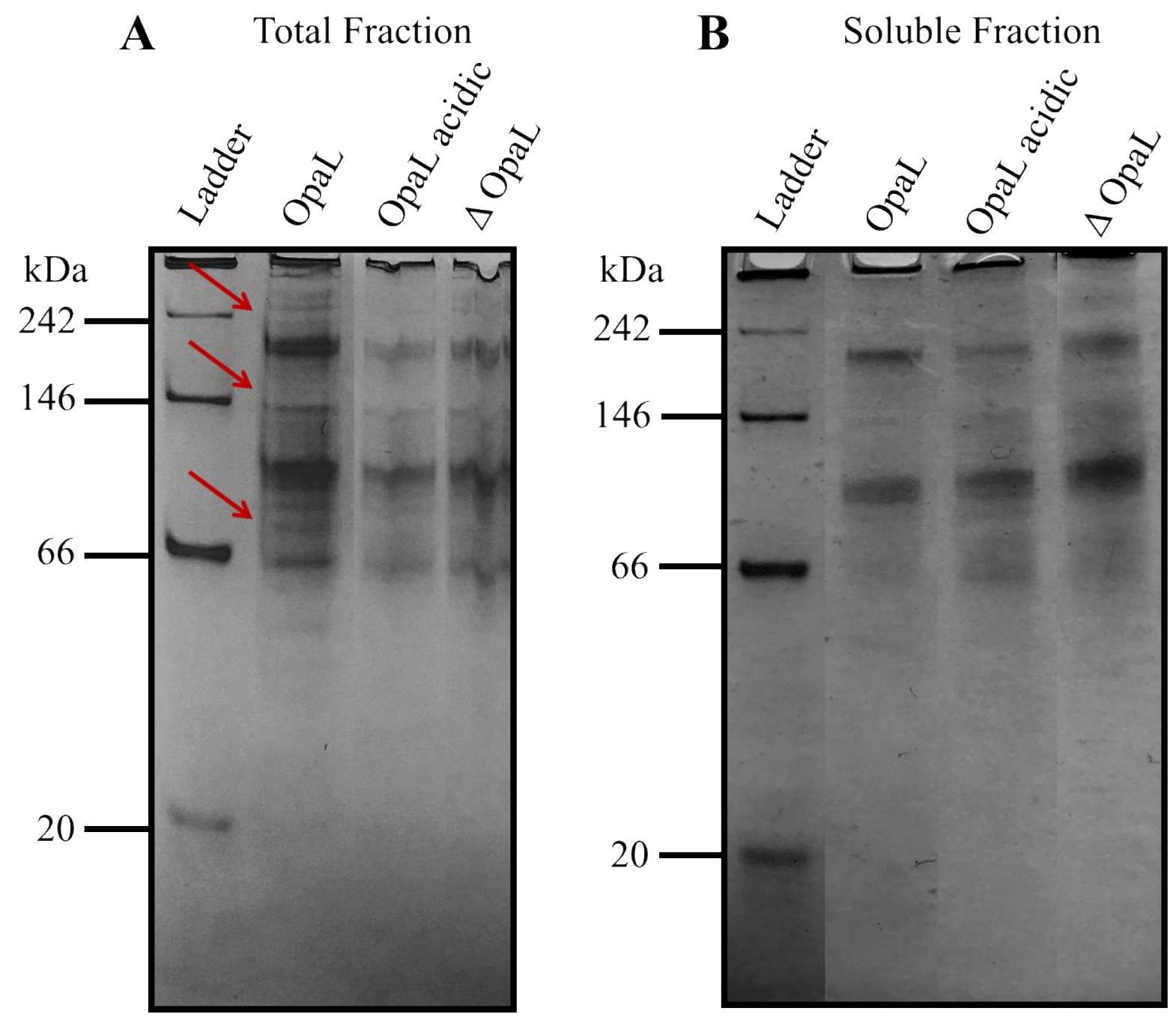

Supplementary Figure S3. Protein extracted from OpaL, OpaLacidic, and $\triangle \mathrm{OpaL}$ run on $8 \%$ Tris-Glycine polyacrylamide gels. (A) Total fraction of extracted protein. (B) Soluble fraction of extracted protein. Arrows in panel A indicate bands corresponding to aggregated insoluble proteins not observed in the soluble fraction of proteins shown in panel B. The number and abundance of aggregated protein bands is highest for OpaL strain (panel A). The soluble fraction of proteins is similar across OpaL, OpaLacidic and $\Delta$ OpaL. These results support that OpaL causes formation of aggregated proteins. 


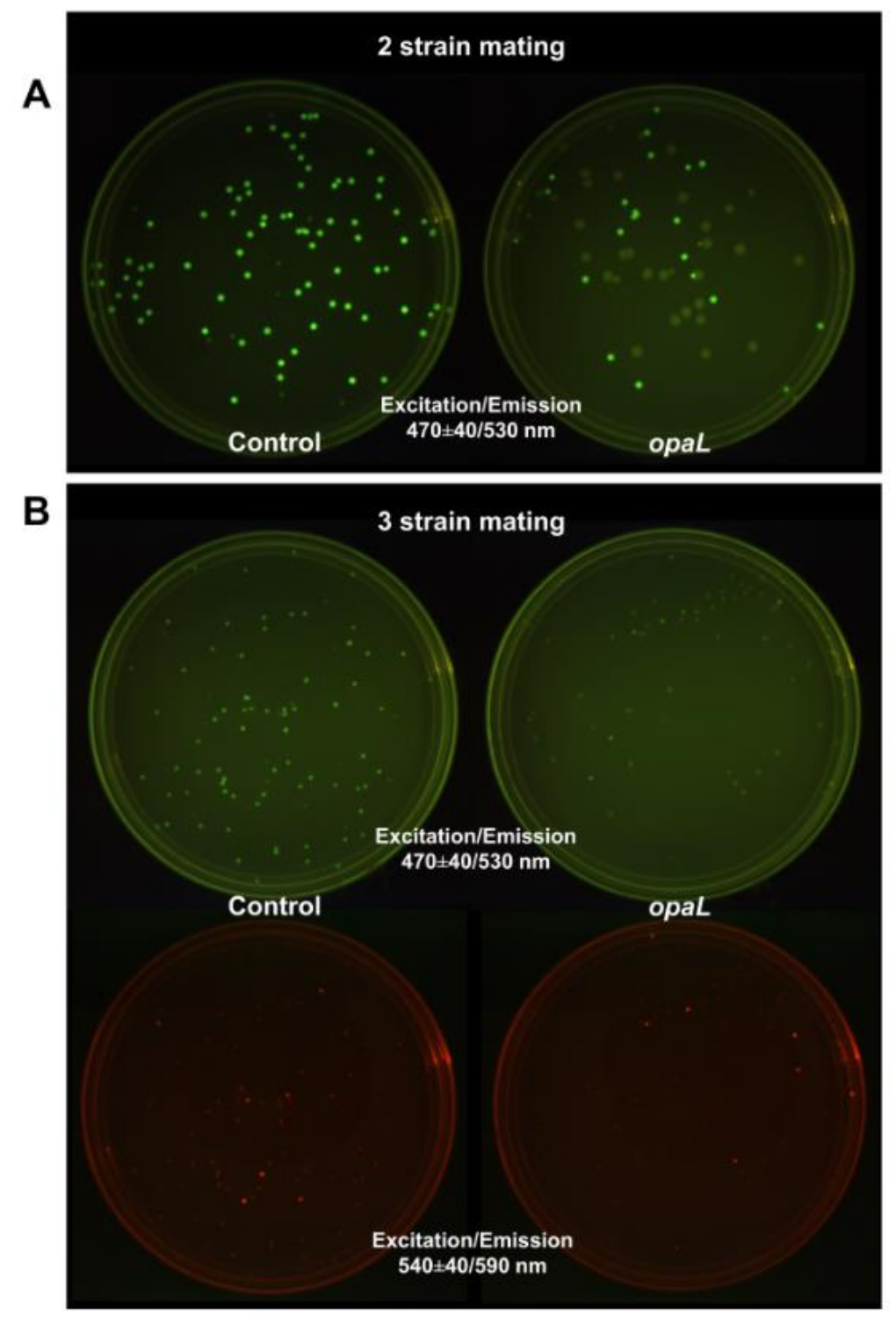

Supplementary Figure S4. (A) One of the replicates used to determine CFUs for the two strain mating-toxicity experiment. (B) One of the replicates used to determine CFUs for the three strain mating-toxicity experiment. The same pair of plates is shown under two different excitation/emission filters to distinguish between GFP expressing recipient $E$. coli BL21 (DE3) and mCherry expressing recipient E. coli $\mathrm{DH} 5 \alpha$. 
Supplementary Table S1. Strains, plasmids, experiments in which each strain was utilized, and references for the sources from which these materials were obtained.

\begin{tabular}{|c|c|c|}
\hline Strains and Plasmids & Experiments & Reference/Source \\
\hline$\overline{E . ~ c o l i ~ C 600, ~ R K 2 ~}$ & $\begin{array}{l}\text { Donors for mating-toxicity assays } \\
\text { and mating assay }\end{array}$ & $\mathrm{ATCC}^{\circledR} 37125^{\mathrm{TM}}$ \\
\hline $\begin{array}{l}\text { E. coli C600, RK2, pET11a- } \\
\text { opaL }\end{array}$ & $\begin{array}{l}\text { Donors for mating-toxicity assays } \\
\text { and mating assay }\end{array}$ & This study \\
\hline E. coli XL1 Blue, pHL662 & Recipients for mating assay & $\begin{array}{l}\text { Adamson and Lim } \\
2013\end{array}$ \\
\hline E. coli BL21 (DE3), pHL662 & $\begin{array}{l}\text { Recipients for mating-toxicity } \\
\text { assays }\end{array}$ & This study \\
\hline $\begin{array}{l}\text { E. coli BL21 (DE3), pET11a- } \\
\text { opaL }\end{array}$ & $\begin{array}{l}\text { Experimental host for toxicity } \\
\text { assay }\end{array}$ & This study \\
\hline $\begin{array}{l}\text { E. coli BL21 (DE3), pET11a- } \\
\text { opaLacidic }\end{array}$ & Control host for toxicity assay & This study \\
\hline $\begin{array}{l}\text { E. coli BL21 (DE3), pET11a- } \\
\text { AopaL }\end{array}$ & $\begin{array}{l}\text { Control host for toxicity assay and } \\
\text { nile red aggregation assay }\end{array}$ & This study \\
\hline E. coli BL21 (DE3) & $\begin{array}{l}\text { Control cells for toxicity assay and } \\
\text { nile red aggregation assay }\end{array}$ & NEB\#C2527I \\
\hline E. coli $\mathrm{DH} 5 \alpha$, pUV145 & $\begin{array}{l}\text { Non-target recipients for three } \\
\text { strain mating-toxicity assay }\end{array}$ & Bordoy et al. 2016 \\
\hline
\end{tabular}

\section{Acknowledgements}

This work has been supported by National Science Foundation award number MCB1714564 to AC. PO and CMC were supported by National Science Foundation Fellowships. The imaging work was performed at the BioFrontiers Institute Advanced Light Microscopy Core with the help of Joe Dragavon. Laser scanning confocal microscopy was performed on a Nikon A1R microscope 
supported by NIST-CU Cooperative Agreement award number 70NANB15H226. The funders had no role in study design, data collection and analysis, or preparation of the manuscript.

\section{References}

(1) Kumarasamy, K. K.; Toleman, M. A.; Walsh, T. R.; Bagaria, J.; Butt, F.; Balakrishnan, R.; Chaudhary, U.; Doumith, M.; Giske, C. G.; Irfan, S.; et al. Emergence of a New Antibiotic Resistance Mechanism in India, Pakistan, and the UK: A Molecular, Biological, and Epidemiological Study. Lancet. Infect. Dis. 2010, 10 (9), 597-602.

(2) Stefani, M.; Dobson, C. M. Protein Aggregation and Aggregate Toxicity: New Insights into Protein Folding, Misfolding Diseases and Biological Evolution. J. Mol. Med. (Berl). 2003, 81 (11), 678-699.

(3) Bednarska, N. G.; van Eldere, J.; Gallardo, R.; Ganesan, A.; Ramakers, M.; Vogel, I.; Baatsen, P.; Staes, A.; Goethals, M.; Hammarström, P.; et al. Protein Aggregation as an Antibiotic Design Strategy. Mol. Microbiol. 2016, 99 (5), 849-865.

(4) Khodaparast, L.; Khodaparast, L.; Gallardo, R.; Louros, N. N.; Michiels, E.;

Ramakrishnan, R.; Ramakers, M.; Claes, F.; Young, L.; Shahrooei, M.; et al. Aggregating Sequences That Occur in Many Proteins Constitute Weak Spots of Bacterial Proteostasis. Nat. Commun. 2018, 9 (1), 866.

(5) Fernandez-Escamilla, A.-M.; Rousseau, F.; Schymkowitz, J.; Serrano, L. Prediction of Sequence-Dependent and Mutational Effects on the Aggregation of Peptides and Proteins. Nat. Biotechnol. 2004, 22 (10), 1302-1306.

(6) Ingram, L. C.; Richmond, M. H.; Sykes, R. B. Molecular Characterization of the R Factors Implicated in the Carbenicillin Resistance of a Sequence of Pseudomonas 
Aeruginosa Strains Isolated from Burns. Antimicrob. Agents Chemother. 1973, 3 (2), 279288.

(7) Waters, V. L. Conjugation between Bacterial and Mammalian Cells. Nat Genet 2001, 29 (4), 375-376.

(8) Guiney, D. G. Host Range of Conjugation and Replication Functions of the Escherichia Coli Sex Plasmid Flac. Comparison with the Broad Host-Range Plasmid RK2. J. Mol. Biol. 1982, 162 (3), 699-703.

(9) Guiney, D. G.; Yakobson, E. Location and Nucleotide Sequence of the Transfer Origin of the Broad Host Range Plasmid RK2. Proc. Natl. Acad. Sci. U. S. A. 1983, 80 (12), 35953598.

(10) Adamczyk, M.; Jagura-Burdzy, G. Spread and Survival of Promiscuous IncP-1 Plasmids. Acta Biochim. Pol. 2003, 50 (2), 425-453.

(11) Lee, C.; Kim, J.; Shin, S. G.; Hwang, S. Absolute and Relative QPCR Quantification of Plasmid Copy Number in Escherichia Coli. J. Biotechnol. 2006, 123 (3), 273-280.

(12) Thomas, C. M.; Cross, M. a; Hussain, a a; Smith, C. a. Analysis of Copy Number Control Elements in the Region of the Vegetative Replication Origin of the Broad Host Range Plasmid RK2. EMBO J. 1984, 3 (1), 57-63.

(13) Cook, L.; Chatterjee, A.; Barnes, A.; Yarwood, J.; Hu, W.-S.; Dunny, G. Biofilm Growth Alters Regulation of Conjugation by a Bacterial Pheromone. Mol. Microbiol. 2011, 81 (6), $1499-1510$.

(14) Hoiby, N.; Ciofu, O.; Bjarnsholt, T. Pseudomonas Aeruginosa Biofilms in Cystic Fibrosis. Future Microbiol. 2010, 5 (11), 1663-1674.

(15) Hausner, M.; Wuertz, S. High Rates of Conjugation in Bacterial Biofilms as Determined 
by Quantitative in Situ Analysis. Appl. Environ. Microbiol. 1999, 65 (8), 3710-3713.

(16) Citorik, R. J.; Mimee, M.; Lu, T. K. Sequence-Specific Antimicrobials Using Efficiently Delivered RNA-Guided Nucleases. Nat Biotech 2014, 32 (11), 1141-1145.

(17) Ji, W.; Lee, D.; Wong, E.; Dadlani, P.; Dinh, D.; Huang, V.; Kearns, K.; Teng, S.; Chen, S.; Haliburton, J.; et al. Specific Gene Repression by CRISPRi System Transferred through Bacterial Conjugation. ACS Synth. Biol. 2014, 3 (12), 929-931.

(18) Bikard, D.; Euler, C.; Jiang, W.; Nussenzweig, P. M.; Goldberg, G. W.; Duportet, X.; Fischetti, V. A.; Marraffini, L. A. Development of Sequence-Specific Antimicrobials Based on Programmable CRISPR-Cas Nucleases. Nat. Biotechnol. 2014, 32 (11), 11461150.

(19) Peng, Y.; Rakowski, S. A.; Filutowicz, M. Small Deletion Variants of the Replication Protein, Pi, and Their Potential for over-Replication-Based Antimicrobial Activity. FEMS Microbiol. Lett. 2006, 261 (2), 245-252.

(20) Filutowicz, M.; Burgess, R.; Gamelli, R. L.; Heinemann, J. A.; Kurenbach, B.; Rakowski, S. A.; Shankar, R. Bacterial Conjugation-Based Antimicrobial Agents. Plasmid 2008, 60 (1), 38-44.

(21) Schwartz, R.; Istrail, S.; King, J. Frequencies of Amino Acid Strings in Globular Protein Sequences Indicate Suppression of Blocks of Consecutive Hydrophobic Residues. Protein Sci. 2008, 10 (5), 1023-1031.

(22) Turoverov, K. K.; Kuznetsova, I. M.; Uversky, V. N. The Protein Kingdom Extended: Ordered and Intrinsically Disordered Proteins, Their Folding, Supramolecular Complex Formation, and Aggregation. Prog. Biophys. Mol. Biol. 2010, 102 (2), 73-84.

(23) Bertoncini, C. W.; Jung, Y.-S.; Fernandez, C. O.; Hoyer, W.; Griesinger, C.; Jovin, T. M.; 
Zweckstetter, M. Release of Long-Range Tertiary Interactions Potentiates Aggregation of Natively Unstructured $\alpha$-Synuclein. Proc. Natl. Acad. Sci. U. S. A. 2005, 102 (5), 1430 LP-1435.

(24) Ramshini, H.; Parrini, C.; Relini, A.; Zampagni, M.; Mannini, B.; Pesce, A.; Saboury, A. A.; Nemat-Gorgani, M.; Chiti, F. Large Proteins Have a Great Tendency to Aggregate but a Low Propensity to Form Amyloid Fibrils. PLoS One 2011, 6 (1), e16075.

(25) Wickner, S.; Maurizi, M. R.; Gottesman, S. Posttranslational Quality Control: Folding, Refolding, and Degrading Proteins. Science 1999, 286 (5446), 1888-1893.

(26) Tobias, J. W.; Shrader, T. E.; Rocap, G.; Varshavsky, A. The N-End Rule in Bacteria. Science 1991, 254 (5036), 1374-1377.

(27) Ross, C. A.; Poirier, M. A. Protein Aggregation and Neurodegenerative Disease. Nat. Med. 2004, 10, S10.

(28) Bednarska, N. G.; Schymkowitz, J.; Rousseau, F.; Van Eldere, J. Protein Aggregation in Bacteria: The Thin Boundary between Functionality and Toxicity. Microbiology 2013, 159 (Pt 9), 1795-1806.

(29) Demeule, B.; Gurny, R.; Arvinte, T. Detection and Characterization of Protein Aggregates by Fluorescence Microscopy. Int. J. Pharm. 2007, 329 (1-2), 37-45.

(30) Pansegrau, W.; Lanka, E.; Barth, P. T.; Figurski, D. H.; Guiney, D. G.; Haas, D.; Helinski, D. R.; Schwab, H.; Stanisich, V. A.; Thomas, C. M. Complete Nucleotide Sequence of Birmingham IncPa Plasmids. J. Mol. Biol. 1994, 239 (5), 623-663.

(31) Bordoy, A. E.; Varanasi, U. S.; Courtney, C. M.; Chatterjee, A. Transcriptional Interference in Convergent Promoters as a Means for Tunable Gene Expression. ACS Synth. Biol. 2016, 5 (12), 1331-1341. 
(32) Romero, P. A.; Arnold, F. H. Exploring Protein Fitness Landscapes by Directed Evolution. Nat. Rev. Mol. Cell Biol. 2009, 10 (12), 866-876.

(33) Adamson, D. N.; Lim, H. N. Rapid and Robust Signaling in the CsrA Cascade via RNAProtein Interactions and Feedback Regulation. Proc. Natl. Acad. Sci. U. S. A. 2013, 110 (32), 13120-13125.

(34) Baneyx, F. Recombinant Protein Expression in Escherichia Coli. Curr. Opin. Biotechnol. 1999, $10(5), 411-421$.

(35) Wilks, J. C.; Slonczewski, J. L. PH of the Cytoplasm and Periplasm of Escherichia Coli: Rapid Measurement by Green Fluorescent Protein Fluorimetry . Journal of Bacteriology. August 2007, pp 5601-5607.

(36) Smith, C. A. Physiology of the Bacterial Cell. A Molecular Approach. By F C Neidhardt, J L Ingraham and M Schaechter. Pp 507. Sinauer Associates, Sunderland, MA. 1990. \$43.95 ISBN 0-87893-608-4; Headington Hill Hall, 1992; Vol. 20.

(37) Xu, D.; Zhang, Y. Ab Initio Protein Structure Assembly Using Continuous Structure Fragments and Optimized Knowledge-Based Force Field. Proteins Struct. Funct. Bioinforma. 2012, 80 (7), 1715-1735.

(38) Guex, N.; Peitsch, M. C. SWISS-MODEL and the Swiss-Pdb Viewer: An Environment for Comparative Protein Modeling. Electrophoresis 1997, 18 (15), 2714-2723.

(39) de la Cruz, F.; Frost, L. S.; Meyer, R. J.; Zechner, E. L. Conjugative DNA Metabolism in Gram-Negative Bacteria. FEMS Microbiol. Rev. 2010, 34 (1), 18-40. 